\title{
POINT PREVALENCE STUDY OF ANTIBIOTIC USE IN A PAEDIATRIC HOSPITAL IN ITALY
}

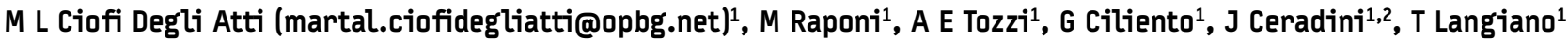 \\ 1. Bambino Gesù Children's Hospital, Rome, Italy \\ 2. Department of Public Health Sciences, University La Sapienza, Rome, Italy
}

A survey aimed to describe the prevalence of antibiotic use in hospitalised children was conducted in June 2007, in Bambino Gesù Children's Hospital in Rome which has the highest annual number of inpatients among paediatric hospitals in Italy. Data were collected by reviewing medical charts of all patients hospitalised for $>48$ hours. A total of 412 hospitalised children were evaluated; their median age was 42.3 months, and $55.6 \%$ were males. Antibiotics were prescribed to 181 of the 412 patients (43.9\%). The prevalence was lowest $(37.7 \%)$ in medical wards, higher (51.1\%) in intensive care units and highest $(52.2 \%)$ in surgical wards. Of the patients treated with antibiotics in surgical wards, $71 \%$ received the treatment as prophylaxis. The most frequently prescribed antibiotics were ceftazidime and the combination of amoxicillin and clavulanic acid. The observed prevalence of antibiotic use was within the range recently reported from other paediatric hospitals in Europe; however, it is advisable to collect data from all over the country in order to identify priority areas and design interventions. These results also highlight the need to implement guidelines for surgical prophylaxis in children, and to further investigate reasons for prescription of parenteral antibiotic therapy in paediatric hospitals.

\section{Introduction}

Antibiotics are among the drugs most commonly prescribed for children. In Italy it has been estimated that $40-50 \%$ of children below 15 years of age receive at least one outpatient antibiotic prescription per year $[1,2]$.

Although the vast majority of antibiotics are consumed in primary care [3], the pressure to select antimicrobial drugs in hospitals appears to be even higher than in outpatient care [4]. An estimated proportion of $36-49 \%$ of hospitalised infants and children receive antibiotics [5-9]. The frequent use of antibiotics is considered to be one of the main reasons for the high prevalence of antimicrobial resistance observed in hospitals [10]. Adverse drug events and excessive costs of treatment are also reasons for concern $[8,11]$, particularly considering that $15-45 \%$ of antibiotic treatment regimens for paediatric patients may be inappropriate $[6,12,13]$.

Surveillance of antimicrobial use in hospitals is therefore important to identify prescribing trends, to link results with antimicrobial resistance data, and to identify areas for improvement.

In this study, we present the results of a survey conducted in 2007 to describe the prevalence of antibiotic use in hospitalised children in Italy. Data have been collected in Bambino Gesù
Children's Hospital in Rome, which is the paediatric hospital with the highest annual number of inpatients in Italy.

\section{Materials and methods \\ Description of the hospital}

Bambino Gesù Children's Hospital is one of the nine children's hospitals in Italy. It is a research hospital within the National Healthcare System and includes two different sites, one located in Rome and the other in Palidoro on the sea coast north of Rome. It is organised in 13 departments and has a total of 607 inpatient bed capacity (444 in Rome and 163 in Palidoro).

In 2007, there were 33,050 hospital inpatient admissions, with a mean length of stay of 5.3 days. The mean number of monthly admissions was 2,738, ranging from 2,016 in August to 3,049 in March. In June, there were 2,893 inpatient admissions.

\section{Population under study}

The point prevalence study was conducted in all hospital departments from 4 to 16 June 2007. Data on antibiotic use were collected by reviewing medical charts of all patients hospitalised for $>48$ hours. For each hospitalised child, information was collected on age, sex, main diagnosis at admission and the type and number of antibiotics administered. Data was also recorded on whether the antimicrobial drugs were prescribed on the basis of clinical signs suggestive of infection, but without microbiological confirmation (i.e. on an empirical basis), or administered for infections that were laboratory confirmed (i.e. based on microbiological findings), or related to prophylaxis.

The antibiotic prescription rates were calculated for the entire hospital and by type of unit, i.e. intensive care units (ICUs), surgical wards and medical wards, including all non-surgical wards apart from ICUs.

\section{Statistics}

Statistical analyses were conducted using STATA 8.2 (Stata Corporation, College Station, Texas, USA).

Differences in rates between groups were compared using the chi-square test or Fisher's exact test; t-test or Mann-Whitney nonparametric test were used to compare continuous variables.

Results

A total of 412 hospitalised children were evaluated; their median age was 42.3 months (range $0-806$ months), and 229 were males (55.6\%). Antibiotics were prescribed for 181 of the 412 patients 
(43.9\%). The prevalence of antibiotic use was higher in older children, ranging from $33.7 \%$ in 0-6-month-old infants (32/95) to $42.4 \%$ in children aged from seven months to five years $(61 / 144)$ and $49.1 \%$ in children older than five years (85/173) (chi-square for trend: $p=0.049$ ). No statistically significant differences by sex were noted.

Out of the total 412 children, 236 were hospitalised in medical wards, 129 in surgical wards and 47 in ICUs. The median age of patients differed significantly, being lowest in ICUs and highest in surgical wards (Table 1 ). The prevalence of antibiotic use was $37.7 \%$ in medical wards, $51.1 \%$ in ICUs and $52.2 \%$ in surgical wards (Table 1). Prevalence by diagnosis at admission is shown in Table 2.

Of the 181 children who were treated with antibiotics, $78(43.8 \%)$ received more than one drug. The prevalence of combination therapy was thus $18.9 \%$.

The total number of antibiotic courses was 255 , i.e. a mean of 1.4 drugs per treated child.

As shown in Figure 1, the top five ranking antibiotics were amoxicillin in combination with clavulanic acid, ceftazidime, ceftriaxone and amikacin.

Antibiotics were prescribed empirically in $51.0 \%$ of cases; in $40.8 \%$ of cases the drugs were used for prophylaxis, and in $8.2 \%$ of cases the treatment was based on microbiological data (Table 3 ).

F I G U R E 1

Number of prescriptions by antibiotic drug, Bambino Gesù Children's Hospital, Rome, Italy, June 2007

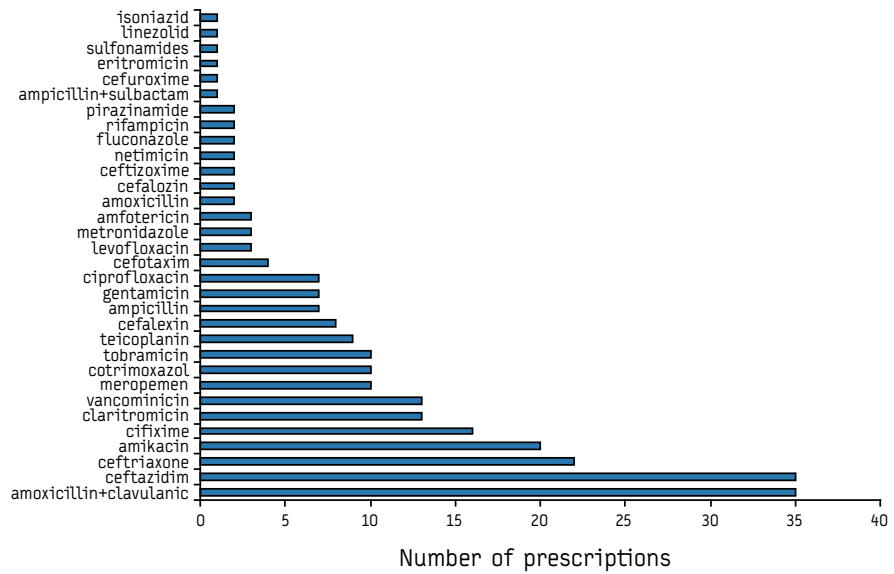

TA B L E 2

Prevalence of antibiotic use, by diagnosis at admission, Bambino Gesù Children's Hospital, Rome, Italy, June 2007

\begin{tabular}{|l|c|c|}
\hline Diagnosis at admission & $\begin{array}{c}\text { Number of } \\
\text { patients }\end{array}$ & $\begin{array}{c}\text { Number of } \\
\text { patients receiving } \\
\text { antibiotics (\%) }\end{array}$ \\
\hline $\begin{array}{l}\text { Symptoms, signs and ill-defined } \\
\text { conditions }\end{array}$ & 72 & $33(45.8)$ \\
\hline Congenital malformations & 48 & $22(45.8)$ \\
\hline Diseases of the cardiovascular system & 43 & $11(25.6)$ \\
\hline Diseases of the respiratory system & 42 & $25(59.5)$ \\
\hline Diseases of the digestive system & 30 & $12(40.0)$ \\
\hline $\begin{array}{l}\text { Diseases of the musculoskeletal } \\
\text { system and connective tissue }\end{array}$ & 22 & $10(45.5)$ \\
\hline $\begin{array}{l}\text { Conditions originating in the perinatal } \\
\text { period }\end{array}$ & 17 & $4(23.5)$ \\
\hline Diseases of the genitourinary system & 16 & $10(62.5)$ \\
\hline Disorders of the nervous system & 13 & $2(15.4)$ \\
\hline Neoplasms & 11 & $8(72.7)$ \\
\hline Injury and poisoning & 11 & $5(45.5)$ \\
\hline Infectious and parasitic diseases & 9 & $7(77.8)$ \\
\hline Diseases of the sense organs & 8 & $6(75)$ \\
\hline Mental disorders & 6 & $0(0)$ \\
\hline $\begin{array}{l}\text { Diseases of the blood and blood- } \\
\text { forming organs }\end{array}$ & 4 & $2(50.0)$ \\
\hline $\begin{array}{l}\text { Endocrine, nutritional and metabolic } \\
\text { diseases, and immune system disorders }\end{array}$ & 4 & $0(0)$ \\
\hline
\end{tabular}

T A B L E 3

Number of prescriptions by antibiotic class and reasons for prescription (microbiological data, clinical data, prophylaxis), Bambino Gesù Children's Hospital, Rome, Italy, June 2007

\begin{tabular}{|l|c|c|c|c|}
\hline Antibiotic class & $\begin{array}{c}\text { Microbiological } \\
\text { data (\%) }\end{array}$ & $\begin{array}{c}\text { Clinical data } \\
(\%)\end{array}$ & $\begin{array}{c}\text { Prophylaxis } \\
(\%)\end{array}$ & Total \\
\hline cephalosporins & $2(2.2)$ & $40(44.4)$ & $48(53.4)$ & 90 \\
\hline penicillins & $5(11.4)$ & $23(52.3)$ & $16(36.3)$ & 44 \\
\hline aminoglycosides & $5(12.8)$ & $18(46.2)$ & $16(41.0)$ & 39 \\
\hline macrolides & $0(0)$ & $13(93.0)$ & $1(7.0)$ & 14 \\
\hline vancomycin & $1(7.0)$ & $12(86.0)$ & $1(7.0)$ & 14 \\
\hline carbapenems & $1(10.0)$ & $7(70.0)$ & $2(20.0)$ & 10 \\
\hline Others & $7(16.0)$ & $17(39.0)$ & $20(45.0)$ & 44 \\
\hline Total & $21(8.2)$ & $130(51.0)$ & $104(40.8)$ & 255 \\
\hline
\end{tabular}

T A B L E

Prevalence of antibiotic use, by basis for prescription (microbiological data, clinical data or prophylaxis), and by type of ward, Bambino Gesù Children's Hospital, Rome, Italy, June 2007

\begin{tabular}{|c|c|c|c|c|}
\hline & Medical ward & Surgical ward & $\begin{array}{c}\text { Intensive care } \\
\text { units (ICUs) }\end{array}$ & p-value \\
\hline Number of patients & 236 & 129 & 47 & - \\
\hline Patients' median age in months (range) & $36.8(0-512)$ & $68.7(0-807)$ & $2.6(0-222)$ & $<0.001$ \\
\hline Number of patients receiving antibiotics based on microbiological data (\%) & $5(2.1)$ & $1(0.07)$ & $6(12.7)$ & $<0.001$ \\
\hline Number of patients receiving antibiotics based on clinical data (\%) & $65(27.5)$ & $19(14.7)$ & $9(19.1)$ & n.s. \\
\hline Number of patients receiving antibiotics for prophylaxis (\%) & $19(8.0)$ & $48(37.2)$ & $9(19.1)$ & $<0.001$ \\
\hline Total number of patients receiving antibiotics (\%) & $89(37.7)$ & $68(52.7)$ & $24(51.1)$ & 0.013 \\
\hline
\end{tabular}


The use of cephalosporins was almost evenly distributed between empirical therapy and prophylaxis, while penicillins were most frequently used for empirical therapy.

Penicillins and aminoglycosides were the two categories of drugs that were most commonly prescribed on the basis of microbiological data.

The highest proportion of children receiving antibiotics prescribed on the basis of microbiological data was found in ICUs $(25.0 \%$ vs. $5.7 \%$ and $1.5 \%$ in medical and surgical wards, respectively; $\mathrm{p} \leq 0.01$ ), while medical wards ranked first in proportion of empirical treatments $(73.0 \%$ vs. $37.5 \%$ in ICUs and $27.9 \%$ in surgical wards; $p<0.01)$, and surgical wards in prophylactic use $(70.6 \%$ vs. $37.5 \%$ in ICUs and $21.3 \%$ in medical wards; $p<0.01$ ).

\section{Discussion}

In 2005, Italy ranked third among European countries with the highest consumption of antibiotics in outpatient care [14], and a recent literature review of studies published in USA, Canada, north-central Europe and Italy found that Italy also has one of the highest paediatric outpatient antibiotic prescription rates [15]. Although a strong positive correlation between the extent of antibiotic consumption in outpatient and inpatient care has been shown [4], no national data on hospital consumption have been collected in Italy up to now, and no national policies on the prudent use of antibiotic have been implemented.

In western Europe, studies on hospital use of antibiotics in children are few $[5,6,9]$. In comparison with these findings, our results show higher prevalence of antibiotic use than those observed in the Netherlands and Switzerland in the late 1990s and early 2000 s where prevalence rates were $36 \%[5,6]$, yet lower than those reported from UK in 2006 (49\%) [9]. The proportion of prescriptions that had been based on microbiological data was also similar to that reported by these European surveys.

Our study has some limitations. Firstly, it was conducted in one hospital only, and its results cannot be considered representative of the whole country. Secondly, it was conducted in June, when the number of children admitted with respiratory infections could have been lower than observed in other periods of the year. Since respiratory tract infections are one of the leading causes of antimicrobial use in children [2], we could have underestimated the prevalence. Thirdly, information on the start of antibiotic therapy was not collected, so we cannot exclude the possibility that some children had already been on therapy at admission. Lastly, we did not evaluate the appropriateness of antibiotic prescriptions and we did not investigate if prescriptions were due to nosocomial infections.

In our study, the most frequently used antibiotic was the combination of amoxicillin plus clavulanic acid, as observed in primary care $[1,14]$. This finding confirms that hospital antimicrobial use tends to display a similar distribution pattern to that observed in the ambulatory use [4].

A number of interventions including persuasive and restrictive methods have been shown to be effective in reducing antimicrobial use in hospitals [16]. The commonly prescription pattern observed in hospitalised and outpatient children underscore the need to implement actions targeting both primary care and hospital paediatricians. However, it is well known that health indicators, such as infant mortality rate, vaccination coverage and hospitalisation rates, vary widely across Italy [17]. Variability in outpatient antibiotic prescribing profiles by geographical area has also been shown [18], and it is likely that antibiotic use in children would also differ by hospital. It is therefore advisable to collect data at both hospital and national level, in order to identify priority areas and design interventions tailored to specific circumstances.

Since early 2000s, Bambino Gesù Children's Hospital has implemented a series of measures, including collection of data on antimicrobial resistance, introduction of guidelines for diagnosis and treatment of infectious diseases such as bronchiolitis and acute gastroenteritis, which could have affected the prescribing habits.

An important issue identified in our results is the high proportion of children who received surgical prophylaxis. In fact, $71 \%$ of patients treated with antibiotics in surgical wards received their prescription for prophylaxis, compared to $13-42 \%$ reported by other authors $[6,7]$.

The fact that ceftadizime, a parenteral third-generation cephalosporin, ranked first (together with amoxicillin + clavulanic acid) in prescription frequency is also a reason for concern.

Though we did not evaluate the appropriateness of antibiotic use, these results highlight the need to introduce guidelines for surgical prophylaxis in children, and to further investigate the reasons for prescribing parenteral antibiotic therapy in paediatric hospitals.

\section{References}

1. Resi D, Milandri M, Moro ML; Emilia Romagna Study Group On The Use of Antibiotics In Children. Antibiotic prescriptions in children. J Antimicrob Chemother. 2003;52(2):282-6.

2. Ciofi degli Atti ML, Massari M, Bella A, Boccia D, Filia A, Salmaso S, et al. Clinical, social and relational determinants of paediatric ambulatory drug prescriptions due to respiratory tract infections in Italy. Eur J Clin Pharmacol. 2006;62(12):1055-64.

3. Müller-Pebody B, Muscat M, Pelle B, Klein BM, Brandt CT, Monnet DL. Increase and change in pattern of hospital antimicrobial use, Denmark, 1997-2001. J Antimicrob Chemother. 2004:54(6):1122-6.

4. Vander Stichele RH, Elseviers MM, Ferech M, Blot S, Goossens H, European Surveillance of Antibiotic Comsuption (ESAC) Project Group. Hospital consumption of antibiotics in 15 European countries: results of the ESAC Retrospective Data Collection (1997-2002). J Antimicrob Chemother. 2006;58(1):159-67.

5. van Houten MA, Luinge K, Laseur M, Kimpen JL. Antibiotic utilisation for hospitalised paediatric patients. Int J Antimicrob Agents. 1998;10(2):161-4.

6. Potocki M, Goette J, Szucs TD, Nadal D. Prospective survey of antibiotic utilization in pediatric hospitalized patients to identify targets for improvement of prescription. Infection. 2003;31(6):398-403.

7. Hajdu A, Samodova OV, Carlsson TR, Voinova LV, Nazarenko SJ, Tjurikov $A V$, et al. A point prevalence survey of hospital-acquired infections and antimicrobial use in a paediatric hospital in north-western Russia. J Hosp Infect. 2007;66(4):378-84.

8. Berild D, Abrahamsen TG, Andresen S, Bjørløw E, Haug O, Kossenko IM, et al. A controlled intervention study to improve antibiotic use in a Russian paediatric hospital. Int J Antimicrob Agents. 2008;31(5):478-83.

9. Ang L, Laskar R, Gray JW. A point prevalence study of infection and antimicrobial use at a UK children's hospital. J Hosp Infect. 2008;68(4):372-4.

10. de Man P, Verhoeven BA, Verbrugh HA, Vos MC, van den Anker JN. An antibiotic policy to prevent emergence of resistant bacilli. Lancet. 2000;355(9208):973-8.

11. Shehab N, Patel PR, Srinivasan A, Budnitz DS. Emergency department visits for antibiotic-associated adverse events. Clin Infect Dis 2008:47(6):735-43.

12. Schollenberg E, Albritton WL. Antibiotic misuse in a pediatric teaching hospital. Can Med Assoc J. 1980;122(1):49-52.

13. Principi N, Marchisio P, Sher D, Boccazzi A, Moresco RC, Viola G, Sereni F. Control of antibiotic therapy in paediatric patients. II. Appropriateness of antibiotic choice in selected diseases. Eur J Clin Pharmacol. 1981;20(2):119-21. 
14. Muller A, Coenen S, Monnet DL, Goossens H. European Surveillance of Antimicrobial Consumption (ESAC): outpatient antibiotic use in Europe, 1998-2005. Euro Surveill. 2007;12(41):pii=3284. Available from: http://www. eurosurveillance.org/ViewArticle. aspx?ArticleId $=3284$

15. Rossignoli A, Clavenna A, Bonati M. Antibiotic prescription and prevalence rate in the outpatient pediatric population: analysis of surveys published during 2000-2005. Eur J Clin Pharmacol, 2007;63:1099-1106.

16. Davey P, Brown E, Fenelon L, Finch R, Gould I, Hartman G, et al. Interventions to improve antibiotic prescribing practices for hospital inpatients. Cochrane Database Syst Rev. 2005;(4):CD003543.

17. Osservatorio Nazionale sulla Salute nelle Regioni Italiane. Rapporto Osservasalute 2007. Stato di salute e qualità dell'assistenza nelle Regioni Italiane. Prex, 2007. Available from: http://www.osservasalute.it/

18. Cucinotta G, Mazzaglia G, Toscano MA, Arcoraci V, Tempera G, Salmeri $M$, et al. Exploring the variability in antibiotic prescribing profiles among paediatricians from two different areas of Italy. Pharmacol Res. 2002;45(5):369-74.

This article was published on 9 0ctober 2008

Citation style for this article: Ciofi Degli Atti ML, Raponi M, Tozzi AE, Ciliento G, Ceradin $\mathrm{J}$, Langiano T. Point prevalence study of antibiotic use in a paediatric hospital in Italy. Euro Surveill. 2008;13(41):pii=19003. Available online: http://www.eurosurveillance.org/ ViewArticle.aspx?ArticleId $=19003$ 